\title{
Economic impact of infections among patients with primary immunodeficiency disease receiving IVIG therapy
}

This article was published in the following Dove Press journal:

ClinicoEconomics and Outcomes Research

10 June 2014

Number of times this article has been viewed

Joseph Menzin'

Matthew Sussman'

Michael Munsell'

Arthur Zbrozek ${ }^{2}$

'Boston Health Economics, Inc., Waltham, MA, USA; ${ }^{2}$ CSL Behring, LLC, King of Prussia, PA, USA
Correspondence: Joseph Menzin Boston Health Economics, Inc., 20 Fox Road, Waltham, MA 0245I, USA $\mathrm{Tel}+\mathrm{I} 78 \mathrm{I} 2900808$

Fax + I 78I 2900029

Email jmenzin@bhei.com
Purpose: There are limited data on the cost of infections among patients with primary immunodeficiency disease (PIDD) in clinical practice. The purpose of this study was to assess the economic impact, from the US commercial payer perspective, of infections in a cohort of patients with PIDD who were administered intravenous immunoglobulin (IVIG) therapy.

Methods: This study used administrative claims from the MarketScan ${ }^{\circledR}$ Database. Patients with a PIDD diagnosis, one or more prescription(s) for IVIG therapy between January 1, 2008 and February 28, 2010, and one or more prescription(s) for IVIG at least 3 months following first IVIG prescription, were selected. The study period consisted of a 7-month window following first IVIG prescription. Study measures included infection-related medical resource use and expenditures. Adjusted infection-related hospitalization expenditures were estimated using a generalized linear model, controlling for demographics, comorbidities, and infection type.

Results: A total 1,742 patients with PIDD and consistent IVIG use were identified, with 490 patients (mean age $43 ; 58.8 \%$ female) having one or more infection(s) during the 7-month study period. Infection-related inpatient hospitalizations were the most expensive component of care (US\$38,574 per hospitalized patient). In multivariate modeling, the presence of a blood infection during the hospitalization (versus [vs] no blood infection), having diabetes, and younger age $(<18$ vs 55-64) were associated with significant increases in infection-related hospitalization expenditures $(49.3 \%, 55.3 \%$, and $76.5 \%$, respectively) $(P<0.05)$.

Conclusion: Health care expenditures for infections in PIDD patients receiving IVIG therapy can be substantial, particularly for inpatient care. Future evaluations assessing the incremental cost of optimizing IVIG therapy should include evaluation of the effects on infection-related medical expenditures.

Keywords: immunology, immunoglobulin replacement therapy, outcomes research, economics, treatment, resource utilization

\section{Introduction}

Primary immunodeficiency disease (PIDD) refers to a group of genetic disorders in which essential functions of an individual's immune system are intrinsically impaired. Defects can be inherent in cells such as T lymphocytes, B lymphocytes, or phagocytic cells, consequently inhibiting an individual's ability to produce antibodies or fight infection. As a result, patients with PIDD face an increased susceptibility to infection (ie, prolonged, frequent, or uncommonly severe infections). ${ }^{1}$

Data from a telephone survey conducted by the Immune Deficiency Foundation estimate the prevalence of PIDD to be approximately one in 1,200 individuals in the United States (ie, 250,000 people). ${ }^{2}$ However, the prevalence is believed to be underestimated due to a high rate of underdiagnosis. A cohort study conducted from 
1976 through 2006 showed the incidence of PIDD to be 4.6 per 1,000,000 person-years, with rates increasing in more recent years, due to better diagnostic techniques and awareness among medical providers. ${ }^{3}$ For patients with significantly low immunoglobulin (Ig) production levels (IgG levels of less than $200 \mathrm{mg} / \mathrm{dL}$ ), intravenous Ig (IVIG) therapy is a common and effective treatment for providing increased immunity. ${ }^{4}$ The infused antibodies that are administered by IVIG are naturally metabolized, therefore requiring repeat doses at regular intervals over a patient's lifetime. ${ }^{4}$ The benefits of IVIG therapy are temporary, with IgG levels fluctuating from peak to trough throughout the typical 3- to 4- week dosing interval, and the risk of infection among patients with PIDD can still be prevalent during therapy.

PIDD can have a significant impact on a patient's quality of life, as well as present a substantial economic burden to patients and the health care system. A survey conducted by the Jeffrey Modell Centers Network in 64 countries across six continents found that in the year prior to diagnosis, the average patient with undiagnosed PIDD experienced 70 physician/ emergency room (ER) visits, 19 hospitalizations, and $34 \mathrm{missed}$ days of school/work annually. ${ }^{5}$ These figures improved with proper diagnosis and treatment but still presented a significant burden on patients (eg, 12 ER visits and five hospitalizations annually with proper diagnosis and treatment). The majority of published data on the costs associated with the condition focus on the direct medical expenditures associated with Ig therapy. ${ }^{6,7}$ However, with infections being common among this patient population, even among those receiving IVIG therapy (eg, over two infections per patient per year on average, with some episodes leading to a hospitalization), ${ }^{8}$ it is important to also determine the economic burden associated with chronic infections. Published data on the cost of infections in clinical practice are limited; therefore, this study set out to assess the economic impact - from the US commercial payer perspective - of infections in a cohort of PIDD patients who were administered IVIG therapy. The findings will help to establish a more comprehensive estimate of the economic burden of PIDD to the US health care system.

\section{Methods}

\section{Patient selection}

This retrospective database analysis used administrative medical and pharmacy claims from the Truven Health Analytics MarketScan ${ }^{\circledR}$ Commercial Research Database ${ }^{9}$ between January 1, 2008 and September 30, 2010. The MarketScan ${ }^{\circledR}$ Commercial database is constructed from privately insured paid medical and prescription drug claims from approximately 170 million employees and their dependents since 1995. The medical claims capture details regarding dates of service, place of service, up to four International Classification of Diseases, Ninth Revision, Clinical Modification (ICD-9-CM) diagnosis codes, procedure codes, charges, and health plan payments. Pharmacy claims include details on dispense date, National Drug Code (NDC), quantity of medication dispensed, days supplied, and health plan payments. The eligibility file contains details on monthly medical and pharmacy eligibility, patient age, sex, and geographical region. The database is representative of the medical experience of insured employees and their dependents across all regions of the United States.

Patients were identified on the basis of having a diagnosis of PIDD on a medical claim (ICD-9-CM diagnosis codes: 279.00-279.06, 279.12, or 279.2x) anytime between January 1, 2008 and September 30, 2010. Additionally, patients had to have received at least one prescription for IVIG therapy between January 1, 2008 and February 28, 2010 and received at least one prescription for IVIG at least 3 months following the first IVIG prescription, to confirm consistent IVIG use. For each patient, the study period consisted of a 7-month window following their first IVIG prescription. This time period was chosen to maximize the number of patients in the sample, given the relatively low prevalence of PIDD. Other selection criteria required during the study period included: diagnosis of at least one bacterial and/or nonbacterial/unspecified infection, continuous eligibility, no receipt of a prescription for subcutaneous Ig therapy (ie, in order to ensure the same route of Ig administration for each patient, given differences in the adverse event profiles), ${ }^{4}$ and no diagnoses of immune thrombocytopenic purpura, chronic inflammatory demyelinating polyneuropathy, B cell chronic lymphocytic leukemia, or Kawasaki disease (ie, given that these conditions are also treated by IVIG therapy).

\section{Study measures}

Study patients were described in terms of demographic and clinical characteristics. Patient demographic characteristics included age, sex, metropolitan statistical area, and geographic region. Clinical characteristics included an assessment of infection type and the presence of selected comorbid conditions. Infection types included blood (eg, sepsis), lung (eg, pneumonia, respiratory syncytial virus, etc), other (eg, infectious enteritis, helicobacter pylori, etc), and unspecified viral or bacterial organisms (eg, infections indicated but not otherwise specified).

The primary outcome measures included infectionrelated resource utilization and infection-related medical 
expenditures incurred during the 7-month study window. Resource utilization was measured in terms of inpatient hospitalizations, ER visits, outpatient visits, and other resource use, defined as assisted living facility, skilled nursing facility, independent clinic, state/local public health clinic, and rural health clinic. The proportion of patients using specified services and the frequency of use were assessed. In addition, infection-related medical expenditures were assessed in total and for each component of care, as defined above. Infectionrelated resource utilization and medical expenditures were determined by the presence of an infection diagnosis code (primary or any diagnosis) on a claim. All medical expenditures represent 2010 US dollars.

Since the primary aim of our study was to assess infectionrelated costs associated with the various components of care, data were analyzed at the medical claim level. Data were not further categorized into mutually exclusive infection episodes, which can be challenging using administrative claims data alone. As a result, direct comparisons of the incidence rates for infection cannot be made to the current literature.

\section{Statistical analyses}

Descriptive statistics were assessed for patients' demographic and clinical characteristics as well as for each outcome measure. Continuous variables were summarized using mean, standard deviation (SD), median, and interquartile range values. Binary or categorical variables were summarized using percentages.

Given that hospitalizations have been shown to be a significant component of overall resource utilization for patients with PIDD, multivariate analyses were conducted to determine the predictors of infection-related hospitalization expenditures. ${ }^{5}$ Adjusted infection-related hospitalization expenditures were estimated using a generalized linear model with a negative binomial distribution and a log link, controlling for age, sex, and the presence of selected comorbidities. Due to common characteristics observed in health care cost data, such as a highly skewed distribution from the small percentage of patients with extremely high expense rates, the generalized linear model is highly recommended for models using administrative claims data. ${ }^{10}$ The presence of blood and lung infection types were also controlled for in separate multivariate regression models, as patients may have had more than one infection type. The models were conducted at the hospitalization level, instead of at the patient level as some patients may have had more than one infection-related hospitalization.

All analyses were performed using Statistical Analysis System (SAS) software package, version 9.3 (SAS Institute Inc., Cary, NC, USA).

\section{Results}

A total of 1,742 patients with a diagnosis of PIDD and consistent IVIG use were identified in the database; of these, 490 patients had resource utilization indicative of at least one infection during the 7-month study period. Mean age ( \pm SD) among the 490 patients in the final study sample was 43.0 years $( \pm 18.4)$, and the majority of patients were female $(58.8 \%)$ (Table 1). The most prevalent comorbid conditions in this population included asthma $(29.4 \%)$, the presence of any primary malignancy $(25.1 \%)$, and diabetes with chronic complications (13.5\%). A total of $40.4 \%$ of patients experienced a lung infection during the study period, and $11.8 \%$ of patients experienced a blood infection.

The predominant type of infection-related resource use was outpatient care, with $440(89.8 \%)$ patients having one or more infection-related office visit(s) (mean office visits among patients with an office visit was 2.96 per patient). A further $123(25.1 \%)$ patients experienced an infectionrelated inpatient hospitalization, with a mean of 1.38 hospitalizations per patient (median hospital days: 6.5; interquartile range: 4.0-12.0) among those hospitalized. Only 22 patients (4.5\%) had an infection-related ER visit (not hospitalized) (Table 2).

The mean total infection-related medical expenditures over the 7-month study window were US\$11,925 $( \pm \$ 37,498)$ per patient. Infection-related inpatient hospitalizations were the most expensive component of care, at US\$38,574 per hospitalized patient, followed by outpatient visits (US\$1,460 per patient with an outpatient visit) and ER visits (US\$899 per patient with an ER visit) (Table 2 ). In the first multivariate regression, the presence of a blood infection during the hospitalization (versus [vs] no blood infection), having diabetes, and younger age ( $<18$ vs 55-64 years of age) were associated with significant increases in infection-related hospitalization expenditures (49.3\%, 55.3\%, and 76.5\%, respectively) $(P<0.05)$, among those patients experiencing an infection-related hospitalization (Table 3 ). A second multivariate regression was conducted to test the impact of a lung infection during the hospitalization (vs no lung infection) on infection-related expenditures. While younger age and diabetes were still associated with significant increases in infection-related hospitalization expenditures in this model, the presence of a lung infection during the hospitalization was not a significant predictor (Table 4).

\section{Discussion}

There are limited data on infection-related costs experienced by patients with PIDD, with available economic data focusing 
Table I Sociodemographic and clinical characteristics of patients receiving IVIG and experiencing an infection

\begin{tabular}{ll}
\hline Characteristic & Estimate \\
\hline $\mathrm{N}$ & 490 \\
Age (\%) & \\
0 to 4 & $2.7 \%$ \\
5 to II & $8.0 \%$ \\
I2 to I7 & $5.5 \%$ \\
18 to 34 & $11.2 \%$ \\
35 to 44 & $13.7 \%$ \\
45 to 54 & $23.1 \%$ \\
55 to 64 & $35.9 \%$ \\
Mean & 43.0 \\
SD & 18.4 \\
Median & 50 \\
IQR & $32-57$ \\
Minimum & 1 \\
Maximum & 64 \\
Female (\%) & $58.8 \%$ \\
Metropolitan statistical area (\%) & $84.9 \%$ \\
Geographic region (\%) & \\
West & $41.6 \%$ \\
North Central & $28.0 \%$ \\
South & $16.1 \%$ \\
Northeast & $13.1 \%$ \\
Other & $1.2 \%$ \\
Comorbidities (\%) & \\
Asthma & $29.4 \%$ \\
Any primary malignancy & $25.1 \%$ \\
Diabetes with chronic complications & $13.5 \%$ \\
Rheumatologic disease & $6.1 \%$ \\
Renal failure & $4.3 \%$ \\
Diabetes without chronic complications & $3.9 \%$ \\
AIDS & $11.8 \%$ \\
Metastatic solid tumor & $35.5 \%$ \\
Cystic fibrosis & $32.7 \%$ \\
Hemiplegia or paraplegia & $2.7 \%$ \\
Lung type \\
Blood & $2.2 \%$ \\
Other & $1.4 \%$ \\
Unspecified & $0.6 \%$ \\
\hline Notes Exan & \\
\hline
\end{tabular}

Notes: aExamples of infection types include: blood (eg, sepsis), lung (eg, pneumonia), other (eg, infectious enteritis, helicobacter pylori, etc), unspecified infections (eg, infections indicated but not otherwise specified). A patient could have had more than one type of infection during the study period.

Data source: Truven Health Analytics MarketScan ${ }^{\circledast}$ Commercial Research Database, January I, 2008-September 30, 2010.

Abbreviations: AIDS, acquired immunodeficiency syndrome; IQR, interquartile range; IVIG, intravenous immunoglobulin (therapy); SD, standard deviation.

on all-cause resource use or the direct cost of therapy. $6,7,11$ A recent report from the Jeffrey Modell Foundation estimated annual infection-related charges to be $\$ 18,368$ per patient, among those who received a PIDD diagnosis. ${ }^{5}$ The study used results from a patient survey to determine the average number of infections experienced, among PIDD patients, and then applied the charges associated with the infections
Table 2 Infection-related medical resource use and expenditures over a 7-month period, among patients receiving IVIG and experiencing an infection

\begin{tabular}{ll}
\hline Characteristic & Estimate \\
\hline $\mathrm{N}$ & 490 \\
Inpatient hospitalizations & \\
Number with a hospitalization $(\%)$ & $124(25.3 \%)$ \\
Number of hospitalizations among those hospitalized & \\
Mean $( \pm \mathrm{SD})$ & $1.38(0.80)$ \\
Median & 1.00 \\
IQR & $1.00-1.25$
\end{tabular}

Mean number of hospital days among those hospitalized

$\mathrm{N}$

124

Mean $( \pm \mathrm{SD})$

$10.85(12.61)$

Median

6.50

IQR

$4.00-12.00$

Average expenditures among those hospitalized

Mean $( \pm S D)$

$\$ 38,574(\$ 62,572)$

Median

$\$ 15,667$

IQR

$\$ 9,198-\$ 39,463$

ER visits

Number with an ER visit (\%)

$22(4.5 \%)$

Number of ER visits among those with an ER visit

Mean $( \pm S D)$

Median $\quad 6.00$

IQR

$6.50(3.00)$

$4.00-8.50$

Average expenditures among those with an ER visit

Mean $( \pm$ SD)

$\$ 899(\$ 940)$

Median $\$ 586$

IQR \$239-\$1,I27

Outpatient visits

Number with an office visit (\%) $\quad 440$ (89.8\%)

Number of office visits among those with an office visit

$\begin{array}{ll}\text { Mean }( \pm \mathrm{SD}) & 2.96(4.55) \\ \text { Median } & 2.00 \\ \text { IQR } & 1.00-3.00\end{array}$

Average expenditures among those with an office visit

$\begin{array}{ll}\text { Mean }( \pm S D) & \$ 1,460(\$ 5,353) \\ \text { Median } & \$ 229 \\ \text { IQR } & \$ 103-\$ 649\end{array}$

Other resource use ${ }^{\mathrm{a}}$

Number with other resource use (\%) 70 (14.3\%)

Average expenditures among those with other resource use

$\begin{array}{ll}\text { Mean }( \pm \mathrm{SD}) & \$ 6,182(\$ 17,472) \\ \text { Median } & \$ 628 \\ \text { IQR } & \$ 184-\$ 1,628\end{array}$

Total average medical expenditures

N

Mean $( \pm$ SD) $\quad \$ 11,925(\$ 37,498)$

Median $\$ 444$

IQR \$129-\$7,912

Notes: ${ }^{\circ}$ Other resource use includes nonoffice visit outpatient resource use consisting of: assisted living facility, skilled nursing facility, independent clinic, state/local public health clinic, and rural health clinic, among miscellaneous care types. Total infectionrelated medical expenditures were determined by the presence of an infection diagnosis code (primary or any diagnosis) on any medical claim, and represents the expenditures associated with these infection-related claims. This estimate does not reflect a cost estimate per infection episode.

Data source: Truven Health Analytics MarketScan ${ }^{\circledast}$ Commercial Research Database, January I, 2008-September 30, 2010. All reported expenditures are 2010 US dollars. Abbreviations: ER, emergency room; IQR, interquartile range; IVIG, intravenous immunoglobulin (therapy); SD, standard deviation. 
Table 3 Generalized linear model predicting infection-related hospital expenditures (testing impact of blood infection)

\begin{tabular}{|c|c|c|c|}
\hline Predictor variable & $\begin{array}{l}\text { Parameter } \\
\text { estimate }\end{array}$ & $\begin{array}{l}\text { Standard } \\
\text { error }\end{array}$ & $P$-value \\
\hline Intercept & 9.6938 & 0.1776 & $<0.0001$ \\
\hline \multicolumn{4}{|l|}{ Age group (vs 55-64 years) } \\
\hline $0-17^{\S}$ & $0.5679 \S$ & $0.2234^{\S}$ & $0.011^{\S}$ \\
\hline $18-54$ & 0.2946 & 0.1799 & 0.1015 \\
\hline Male (vs female) & 0.2391 & 0.1507 & 0.1126 \\
\hline Malignancy & -0.1666 & 0.1657 & 0.3147 \\
\hline Diabetes $^{\S}$ & $0.4399 \$$ & $0.1807^{\S}$ & $0.0149 \S$ \\
\hline Asthma & -0.2961 & 0.1774 & 0.0952 \\
\hline Other comorbidities* & 0.0168 & 0.1877 & 0.9286 \\
\hline $\begin{array}{l}\text { Blood infection } \\
\text { (vs no blood infection) }^{\S}\end{array}$ & $0.4009^{\S}$ & $0.1714^{\S}$ & $0.0194^{\S}$ \\
\hline
\end{tabular}

Notes: *Includes rheumatologic disease, renal failure, AIDS, metastatic solid tumor, cystic fibrosis, and hemiplegia; ssignificant $(P<0.05)$.

Data source: Truven Health Analytics MarketScan ${ }^{\circledast}$ Commercial Research Database, January I, 2008-September 30, 2010.

Abbreviations: AIDS, acquired immunodeficiency syndrome; vs, versus.

using data from the Hospital Cost and Utilization Project (HCUP), ${ }^{5}$ which represents hospital billing information. The cost estimate produced in the Modell publication is slightly lower than the annualized infection-related medical costs (ie, payments to providers) observed in our study $(\$ 20,443)$.

Our study is subject to certain limitations that are common to all studies that rely on retrospective claims data, such as potential coding errors and incomplete data. In order to maximize the sample size for analysis, only 7 months of continuous data were required for patient selection. Long-term data for a larger sample of patients with PIDD would be valuable for further economic analysis among this patient population. Furthermore, we were unable to assess infection episodes in

Table 4 Generalized linear model predicting infection-related hospital expenditures (testing impact of lung infection)

\begin{tabular}{|c|c|c|c|}
\hline Predictor variable & $\begin{array}{l}\text { Parameter } \\
\text { estimate }\end{array}$ & $\begin{array}{l}\text { Standard } \\
\text { error }\end{array}$ & $P$-value \\
\hline Intercept & 9.7868 & 0.2206 & $<0.0001$ \\
\hline \multicolumn{4}{|c|}{ Age group (vs 55-64 years) } \\
\hline $0-17$ & $0.7292^{\S}$ & $0.2151^{\S}$ & $0.0007^{\S}$ \\
\hline $18-54^{\S}$ & $0.47^{\S}$ & $0.1693^{\S}$ & $0.0055^{\S}$ \\
\hline Male (vs female) & 0.2081 & 0.1526 & 0.1727 \\
\hline Malignancy & -0.0843 & 0.1625 & $0.604 I$ \\
\hline Diabetes $^{\S}$ & $0.4357^{\S}$ & $0.1846^{\S}$ & $0.0183^{\S}$ \\
\hline Asthma & -0.3583 & 0.1777 & 0.0438 \\
\hline Other comorbidities* & 0.067 & 0.1913 & 0.7263 \\
\hline $\begin{array}{l}\text { Lung infection } \\
\text { (vs no lung infection) }\end{array}$ & -0.0045 & 0.1655 & $0.978 \mathrm{I}$ \\
\hline
\end{tabular}

Notes: *Includes rheumatologic disease, renal failure, AIDS, metastatic solid tumor, cystic fibrosis, and hemiplegia; ssignificant $(P<0.05)$.

Data source: Truven Health Analytics MarketScan ${ }^{\otimes}$ Commercial Research Database, January I, 2008-September 30, 2010.

Abbreviations: AIDS, acquired immunodeficiency syndrome; vs, versus. our study, which may be defined by multiple resource-use events (eg, inpatient hospitalization followed by an outpatient visit may have been grouped into a single infection episode). Instead, we identified resource-use events with any diagnosis code for an infection, in order to analyze infection-related costs according to place of service. Given this methodological limitation, an incidence rate for infections could not be determined. Additionally, IVIG dosing and resulting levels were not available in this data set. This information would have been useful to further define treatment patterns, given that IVIG dosing must be individualized to minimize infection.

The results of our study suggest that health care expenditures for infections in PIDD patients receiving IVIG therapy can be substantial, particularly for inpatient care. The total cost of hospitalizations per patient after diagnosis of PIDD has been estimated at approximately $\$ 8,000$ annually, based on data from patient surveys. ${ }^{5}$ Our analysis provides real-world data on the expenditures of patients with PIDD, a data point that is limited in the published literature. These results will be beneficial for health policy decision makers tasked with evaluating the cost effectiveness of IVIG therapy, which has been estimated at approximately $\$ 30,000$ per patient annually. ${ }^{5}$ Future evaluations to assess the incremental cost of optimizing IVIG therapy (eg, adjusting dosing and/or achieving sustained steady-state levels) should include an evaluation of the effects on infection-related medical expenditures.

\section{Acknowledgment}

This study was funded by CSL Behring, LLC.

\section{Disclosure}

Mr Zbrozek is an employee of the study sponsor. The authors report no other conflicts of interest.

\section{References}

1. Immune Deficiency Foundation. IDF Patient and Family Handbook. Chapter 1: The Immune System and Primary Immunodeficiency Diseases. Towson, MD: Immune Deficiency Foundation; 2008. Available from: URL: http://www.primaryimmune.org/publications/book_pats/e_ch01. pdf. Accessed March 22, 2014.

2. Boyle JM, Buckley RH. Population prevalence of diagnosed primary immunodeficiency diseases in the United States. J Clin Immunol. 2007;27(5):497-502.

3. Joshi AY, Iyer VN, Hagan JB, St Sauver JL, Boyce TG. Incidence and temporal trends of primary immunodeficiency: a population-based cohort study. Mayo Clin Proc. 2009;84(1):16-22.

4. Wasserman RL, Manning SC. Diagnosis and treatment of primary immunodeficiency disease: the role of the otolaryngologist. Am J Otolaryngol. 2011;32(4):329-337.

5. Modell V, Gee B, Lewis DB, et al. Global study of primary immunodeficiency diseases $(\mathrm{PI})$ - diagnosis, treatment, and economic impact: an updated report from the Jeffrey Modell Foundation. Immunol Res. 2011;51(1):61-70. 
6. Connolly M, Simoens S. Kiovig for primary immunodeficiency: reduced infusion and decreased costs per infusion. Int Immunopharmacol. 2011;11(9):1358-1361.

7. Darbà J, Restovic G, Kaskens L, de Agustín T. Direct medical costs of liquid intravenous immunoglobulins in children, adolescents, and adults in Spain. J Clin Pharmacol. 2012;52(4):566-575.

8. Lucas M, Lee M, Lortan J, Lopez-Granados E, Misbah S, Chapel H. Infection outcomes in patients with common variable immunodeficiency disorders: relationship to immunoglobulin therapy over 22 years. JAllergy Clin Immunol. 2010;125(6):1354-1360. e4.

9. Hansen LG, Chang S. Health Research Data for the Real World: The MarketScan Databases. Truven Health Analytics. July, 2012.
10. Blough DK, Ramsey SD. Using generalized linear models to assess medical care costs. Health Serv Outcomes Res Methodol. 2000;1(2): 185-202.

11. Fasth A, Nyström J. Quality of life and health-care resource utilization among children with primary immunodeficiency receiving home treatment with subcutaneous human immunoglobulin. J Clin Immunol. 2008;28(4):370-378.

\section{Publish your work in this journal}

ClinicoEconomics \& Outcomes Research is an international, peerreviewed open-access journal focusing on Health Technology Assessment, Pharmacoeconomics and Outcomes Research in the areas of diagnosis, medical devices, and clinical, surgical and pharmacological intervention. The economic impact of health policy and health systems organization also constitute important areas of coverage. The manuscript management system is completely online and includes a very quick and fair peer-review system, which is all easy to use. Visit http://www.dovepress.com/testimonials.php to read real quotes from published authors.

Submit your manuscript here: http://www.dovepress.com/clinicoeconomics-and-outcomes-research-journal 